\title{
GA-PSO Integration Algorithm and Its Application in Modeling on Furnace Pressure System
}

\author{
Qiming Chen \\ Department of Automation, North China Electric Power University, Baoding 071000, China. \\ 1132181030@qq.com
}

\begin{abstract}
Keywords: particle swarm optimization algorithm, genetic algorithm, system identification, furnace pressure system.
\end{abstract}

\begin{abstract}
PSO algorithm is a kind of swarm intelligence optimization algorithm which has the advantages of simple principle, easy implementation, few parameters needed to adjust and so on. However, the search accuracy of the basic PSO algorithm still needs to be improved. In this paper, a modified PSO algorithm using exponent decline inertia weight is put forward and successfully applied to the parameter identification of the furnace pressure system. This modified PSO algorithm combines the nonlinear optimization and genetic algorithm to optimize the inertia weight and acceleration constants of the basic PSO algorithm, and is proved to be effective in parameter identification.
\end{abstract}

\section{Introduction}

The micro negative pressure of the furnace in thermal power plant is significant for the safety and economic operation of the thermal power plant. If the negative pressure is lower than the limit, the furnace may spit fire and do harm to the equipment and the operator. It will lead to safety accident and have impact on the environmental. On the contrary, if the negative pressure is too high, the efficiency will decrease as the air leakage and the heat loss are increased. [1][2]. In order to design a control system to make the furnace pressure stable in a certain range, the characteristic parameters of the furnace pressure system should be obtained by the system identification firstly.

System identification modeling methods are usually divided into mechanism modeling [3] and experimental modeling. The experimental modeling is suitable for the system which has complex structure and whose internal mechanism is difficult to describe. This modeling mainly includes the step response method, correlation analysis and some intelligent algorithms[4], such as support vector machine[5], neural network[6], genetic algorithm[7],etc. Because of the various factors that influence the furnace pressure of the thermal power plant. The experimental modeling is feasible to identify the parameters of the furnace pressure system. Particle swarm optimization algorithm is a kind of intelligent algorithm which is suitable for parameter identification.

This paper presents a modified particle swarm optimization algorithm which combines genetic algorithm and a mathematical mapping. Simulation shows that the modified algorithm has better performance in convergence and precision. Finally, the modified particle swarm optimization algorithm is used to identify the parameters of the transfer function of the actual furnace pressure system.

\section{PSO AND ITS MODIFICATION}

\subsection{Basic particle swarm optimization algorithm}

PSO is a kind of swarm intelligence optimization algorithm, similar to the process of the birds' foraging. Supposing in a $D$ dimension of the target search space, $m$ particles form a group, with three indicators__ position, speed and fitness value to indicate their characteristics. Each particle searches in this space iteratively. In each process of iteration, the particle updates its own speed and position by the information of the individual optimal value and the global optimal value in accordance with the formula (1) and (2). 


$$
\begin{gathered}
V_{i}(k+1)=\omega_{i}(k) V_{i}(k)+c_{1} r_{1 i}\left(P_{i}(k)-P_{i b}(k)\right)+c_{2} r_{2 i}\left(P_{i}(k)-P_{i g}(k)\right) \\
P_{i}(k+1)=P_{i}(k)+\alpha V_{i}(k+1)
\end{gathered}
$$

The current iteration number is $k . V_{i}$ represents the speed of the particle $i$, whose individual extreme value is $P_{i}$. The group extreme value is $P_{i g}$. The inertia weight is $\omega_{i}$. The acceleration constants are $c_{1}$ and $c_{2}$.

The search performance of the basic particle swarm optimization algorithm has a certain dependence on the parameters, so the search of the global optimal solution cannot be absolutely guaranteed. For the specific problem, the parameter $\omega, c_{1}, c_{2}$ will affect the convergence and the accuracy of the algorithm.

\subsection{Modification of PSO}

After the initialization of the particle swarm, based on the experience $\omega=0.7, c_{1}=1.4, c_{2}=1.4$. Then, build appropriate fitness function according to the specific problem, use genetic algorithm to optimize the three parameters and obtain the inertia factor $\omega$, acceleration constant $c_{1}$ and $c_{2}$. For certain problems, the three parameters obtained, compared with the empirical parameters, make the modified PSO have better global search ability and local search capability.

The local search ability of the classical nonlinear programming algorithm is strong, but the global search ability is weak. Whereas, the genetic algorithm has strong global search ability but its local search ability is generally not satisfactory. In the optimization of the three parameters $\omega, c_{1}, c_{2}$, the GA and nonlinear programming algorithm are combined to enhance the local search ability so that the global optimal solution is more likely to be obtained. In this paper, the GA parameters are set as follows: the population size is 50 , the evolutionary algebra is 200 , the crossover probability is 0.6 , the mutation probability is 0.1 , and a nonlinear optimization is operated every time of evolution of 20 generations.

Using the PSO, this paper adopts dynamic inertia weight algorithm. In the beginning of the iteration, the value of inertia weight $\omega$ is set larger to strengthen the global search ability of PSO. In the later period of iteration, the value of inertia weight $\omega$ is properly decreased to improve the PSO local search ability. Based on the above ideas, this paper presents a new method for calculating the inertia weight of exponential decline(EDIW), as shown in the formula (3).

$$
\omega(k)=\omega_{\text {start }}-\left(\omega_{\text {start }}-\omega_{\text {end }}\right)\left(k / T_{\max }\right)^{e}
$$

In the formula (3), $\omega_{\text {start }}$ is initial inertia weight; $\omega_{\text {end }}$ is minimum inertia weight; $k$ is current iteration number; $T_{\max }$ is maximum iteration number.

Compared to the linearly decreasing inertia weight,the index decreasing inertia weight changes slowly in the beginning of iteration so that the global search ability of the algorithm is better kept. In the later period of iteration, changes quickly so that the local search is more accurate. The change trend of this kind of exponential inertia weight in PSO algorithm is shown in Figure 1.

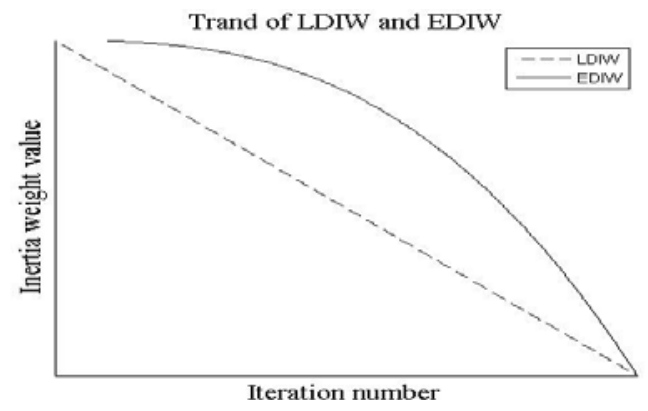

Figure 1 Trend of LDIW and EDIW

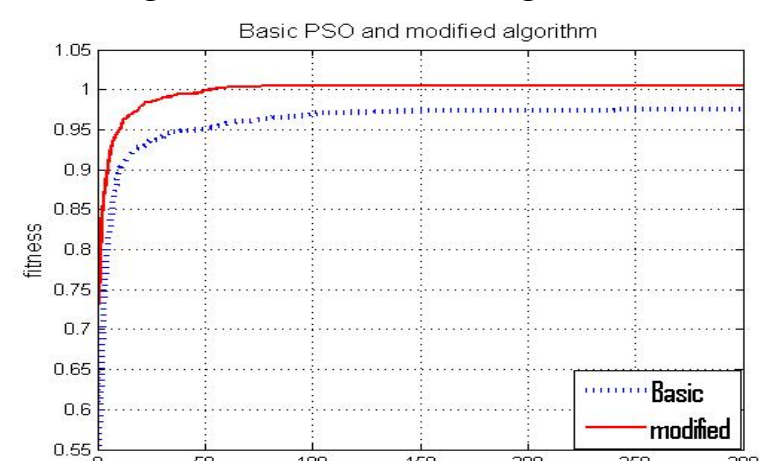

Figure 2 Basic PSO and Modified PSO 
In order to verify the effectiveness of the modified PSO algorithm, we use it to calculate the maximum value of the Mexico Hatnew function in the interval $(0,0)$. The expression of the Mexico Hatnew function is the formula(4).

$$
f(x, y)=\frac{\sin \sqrt{x^{2}+y^{2}}}{\sqrt{x^{2}+y^{2}}}+e^{\frac{\cos 2 \pi x+\cos 2 \pi y}{2}}-2.71289 . x, y \in(-2,2)
$$

To reduce the influence of random errors, the basic PSO algorithm and modified particle swarm optimization algorithm respectively run 100 times. The absolute value of the difference between the average value of the 100 operations and the optimal solution of the Mexico Hat new function is used as the basis for the evaluation of the algorithm's optimization ability. The number of particles in the algorithm is 30, and the number of iteration is 300. Comparison results are shown in figure 2.

The average value of the results of the basic PSO algorithm's 100 operations is 0.9784 , and the error is 0.027. The average value of the modified PSO algorithm's is 0.9959, and the error is 0.0095 . Combined with the curve in figure 2, the author finds that the modified PSO algorithm has a better performance on the accuracy and convergence.

\section{PARAMETER IDENTIFICATION OF FURNACE`S PRESSURE SYSTEM}

In the thermal power plant, the fact that the boiler furnace pressure is maintained in an allowable range plays a significant role for the resource utilization and safe production. In the actual production process, the factors affecting the furnace pressure are various, such as the air blowing, the air exhaust, the coal quality and so on. This paper only identifies the mathematical model of the furnace pressure change caused by the change of the air blowing and the air exhaust. The transfer function between the rest output and input variables can be obtained by the same method.

A 1000MW ultra super-critical thermal power unit operates in $90 \%$ load. After the load changes, the response curve of the change of air blowing, air exhaust and furnace pressure is shown in Figure 3.
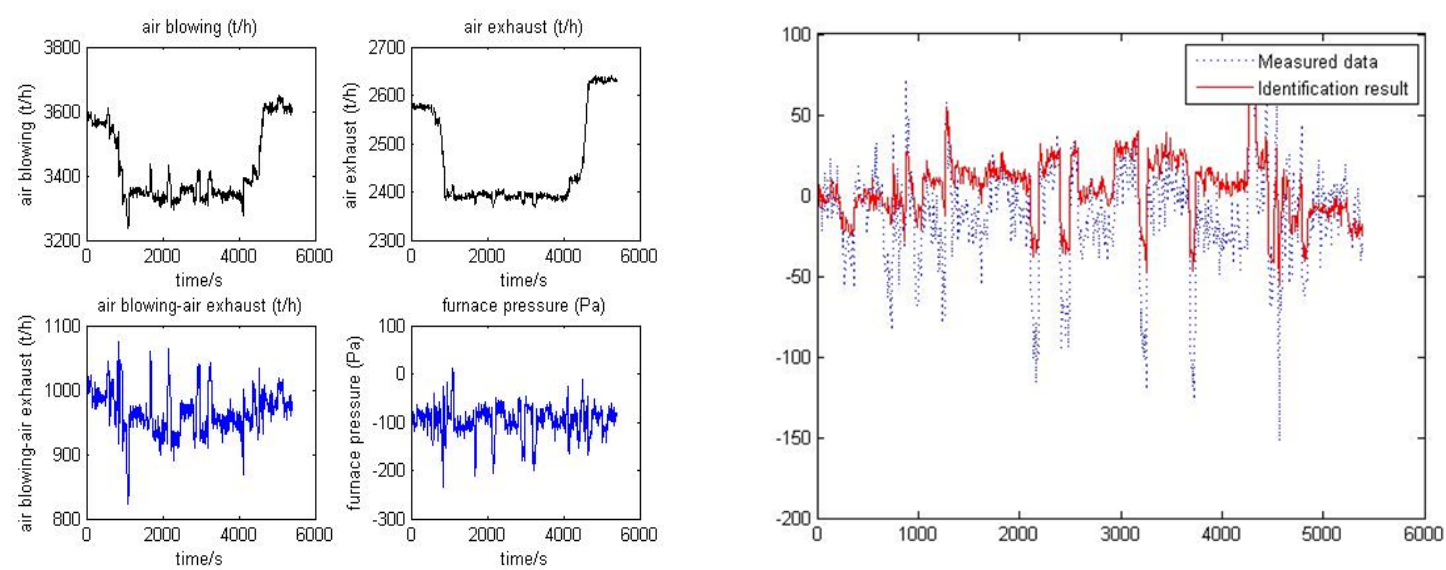

Figure 3Measured data from actual system

Figure 4 Measured data and Simulated data graphs

Through the analysis of the mechanism, we find that if the volume of the air blowing increases, the furnace pressure can be reduced; if the volume of the air exhaust decreases, the furnace pressure also decreases. As seen in the figure 3, the time constant $(T)$ of the furnace pressure control system is small, the system delay is not obvious, and the order of the system (n) is not very high. Combined with the above analysis and the practical experience, in the transfer function selective formula (5) of the furnace pressure system, $K \in(-1,1), T \in(0,5), n \in[1,3]$. In the objective function formula (6), $n$ represents the number of sample data; $y_{i}$ represents the actual system output; $y_{\text {sim }}$ represents the system output obtained by the identification.Algorithm parameter setting: in PSO algorithm, the number of particles is 50 , the number of iterations is 300 , and initially $\omega=0.7, c_{1}=1.4, c_{2}=1.4$; in the GA, parameter settings are the same as 2.2. 


$$
\begin{aligned}
& G(s)=\frac{K}{(T s+1)^{n}} \\
& f=\frac{1}{n} \sum_{i=1}^{n}\left(y_{i}-y_{\text {sim }}\right)^{2}
\end{aligned}
$$

The identification results: $K=0.5793 ; T=2.9737 ; n=2.0999$. The curve of the measured output and the identification result are shown in figure 4.The transfer function of the system is formula (7).

$$
G(s)=\frac{0.5793}{(2.9737 s+1)^{2.0999}}
$$

Because of other disturbances in the measured data, the error of the identification results is acceptable. Combining the results of parameter identification and the curve in figure 4, we consider that the modified particle swarm algorithm proposed in this paper can be applied to practical system identification.

\section{SUMMARY}

PSO algorithm is a great intelligent algorithm, which is easy to implement, universal and has simple principle, but it is still needed to be modified. This paper proposes using genetic algorithm to optimize the inertia weight and the acceleration constant of the basic PSO algorithm and using the exponential decreasing inertia weight to improve the accuracy of the algorithm. Based on the mechanism analysis and the measured data, the modified PSO algorithm is applied to the parameter identification of the actual furnace pressure system. The simulation results show that the modified PSO algorithm is effective.

\section{References}

[1]Gao Yang, Li Jian. Based on EMD-PSO-SVM error correction model of international carbon finance market price prediction [J]. China population, resources and environment, 2014,06:163-170.

[2]Su Chen, Wang Wenlan, Zhang Jiahui, Feng Yongxiang. Study on optimization control of furnace pressure control technology [J]. Huadian strategy system, 2014,11:13-16+77.

[3]Wu Hua. Study on control system of boiler [D]. Harbin Engineering University, 2006

[4]Wang Yongji, Liu Sha, Liu Lei, satellite. Based on the particle swarm optimization algorithm, the online identification method of aerodynamic parameters [J]. Journal of Huazhong University of Science and Technology (NATURAL SCIENCE EDITION), 2016, 03:116-120.

[5]Zhang Ruifeng, Yuan Jingqi. Coal identification technology research and engineering practice of mechanism modeling [J]. Guizhou electric power technology based on 2013,12:7-10.

[6] S. M. JIAO, P. HAN. Chaotic PSO and its application in modeling of circulating fluidized bed boiler combustion system[C]. 2013 IEEE:Conference Anthology. ANTHOLOGY 1-8 Jan. 2013: 1-4

[7]Liu Haifeng. Research on system identification method based on neural network [D]. Xi'an Electronic and Science University, 2007 